\title{
Fortalecimiento de las capacidades de líderes comunales para la gestión local del desarrollo en la Región Huetar Norte
}

Recibido: 29 de agosto, 2019.

Aceptado: 27 de abril, 2020.

Por: M.Sc. Adriana Villalobos Araya ${ }^{1}$, Universidad Estatal a Distancia, Costa Rica, ORCID: 0000-0001-9143-7352

\section{Resumen}

La Universidad Estatal a Distancia (UNED) implementó durante el año 2017 el Técnico en Gestión Local. Esta oferta se enmarca en el reconstruccionismo social, donde el conocimiento se genera a partir del análisis crítico y la resolución de problemas sociales; de forma tal que permita la transformación del sujeto participante y la sociedad en la cual se desenvuelve. El presente documento tiene como objetivo sistematizar dicha experiencia y su contribución en el desarrollo de la Región Huetar Norte, mediante el fortalecimiento de la cultura democrática.

Durante el técnico, se generaron capacidades políticas en líderes comunales de San Carlos, Guatuso, Los Chiles, Upala y San Ramón. Las capacidades alcanzadas por los participantes se categorizan en tres dimensiones: saber teórico, saber hacer y saber ser. Se consideraron como factores de éxito del proceso educativo: la generación de espacios de autoconocimiento y fortalecimiento socioemocional, el análisis de la realidad mediante el uso de metodologías educativas que permitan un proceso de enseñanza aprendizaje contextualizado, la formulación de iniciativas de desarrollo local y la gestión de redes sociales en el territorio.

Como parte del ejercicio de problematización, reflexión con insumos teóricos y aplicación de conocimiento, se logró la formulación de 31 iniciativas de desarrollo local. Dichas iniciativas están orientadas a la gestión en temas ambientales, culturales, productivos, educativos, organizativos, de seguridad, de mobiliario e infraestructura. Para lo cual, se recomienda dar seguimiento a los estudiantes en la ejecución de estas iniciativas, con el fin de valorar su desenvolvimiento en la resolución de problemas sociales y asesorarlos para mejorar su desempeño. En adición, resulta estratégico consolidar la plataforma de la red de gestores locales como catalizador del desarrollo.

1 La profesora Adriana Villalobos Araya es Magister Scientiae en Agricultura Ecológica, graduada en el Centro Agronómico Tropical de Investigación y Enseñanza, Costa Rica. Labora en la Escuela de Ciencias Exactas y Naturales de la Universidad Estatal a Distancia, Costa Rica, como docente, investigadora y coordinadora del Programa de Gestión Local.

Contacto: advillalobos@uned.ac.cr.

PALABRAS CLAVE:

reconstruccionismo social, capacidades políticas, autogestión, desarrollo, trabajo en red

\section{KEY WORDS:}

social reconstructionism, political capacities, self-governance, development, networking 


\section{Abstract}

\section{Boosting community leader capabilities to manage local development in the Huetar Norte region}

In 2017, Universidad Estatal a Distancia (UNED) implemented the Associate Degree in Local Management. Set in the context of social reconstructionism, where knowledge stems from critical analysis and the resolution of social problems, leading to the transformation of both the participating subject and their society. This article seeks to systematize the experience and its contribution to the development of the Huetar Norte region by bolstering democratic culture.

The associate degree fostered policy-making in community leaders in San Carlos, Guatuso, Los Chiles, Upala, and San Ramón. The capacities reached by the participants fit three categories: theoretical knowledge, know-how, and know-howto-be. These are considered success factors in the educational process: generating spaces for self-knowledge and socioemotional empowerment, the analysis of reality by means of educational methodologies that foster learning and teaching in context, design of local development initiatives, and social media management in their territory.

The efforts to address the issue reflect upon theoretical input, and the implementation of knowledge achieved the proposal of 31 local development initiatives, aimed at environment, culture, production, education, and organization management, as well as addressing safety, furnishings, and infrastructure. Students are encouraged to follow up on the execution of these initiatives so they can assess their development in the resolution of social issues and advise them to increase their performance. Additionally, consolidating the local actors' network platform as a catalyst for development.

\section{INTRODUCCIÓN}

Costa Rica goza de una organización democrática, donde, según el Artículo 9 de la Constitución Política, el gobierno lo ejercen el pueblo y tres poderes independientes entre sí; a saber: el poder Legislativo, el Ejecutivo y el Judicial (Ley núm. 8364, 2003). Dicha legislación confiere a la ciudanía el derecho y deber de formar parte en procesos de toma de decisión.

El éxito de la participación ciudadana en la gestión pública depende de que el sistema facilite los espacios y mecanismos para participar, así como, el involucramiento real de actores sociales (Castro y Sibaja, 2016). De acuerdo con LAPOP (citado en Alfaro, 2012), en Costa Rica la participación electoral corresponde a un $66,45 \%$, a pesar de que el voto es obligatorio; y el índice de participación comunal es de un $20,8 \%$.

Según Programa de las Naciones Unidas para el Desarrollo (2013), en Costa Rica la mayor participación se enmarca en formas tradicionales como: los procesos electorales y los procesos administrativos. No obstante, se requiere de una mayor organización civil, donde los participantes se comprometan a ejercer una participación diligente en el análisis y en la búsqueda de soluciones a problemas del entorno. Posteriormente, estas soluciones se plasman en la formulación, la ejecución, el seguimiento y la evaluación de procesos que inciden en el mejoramiento de las condiciones de vida de los habitantes y la defensa de los derechos.

Para lograr un gobierno popular efectivo, la ciudadanía debe contar con capacidades y conocimientos que la faculten para la implementación de transformaciones sociales mediante procesos de participación política, de participación en la vía administrativa y de participación en el entorno local. Con estas capacidades, las personas tendrán voz para definir las normas e instituciones que rigen el país. A su vez, durante la interacción en la sociedad surgen capacidades sociales, las cuales brindan mecanismos que crean oportunidades para concretar proyectos de 
vida y para alcanzar el desarrollo humano (Programa de las Naciones Unidas para el Desarrollo, 2013).

Por tanto, el estado tiene responsabilidad de crear capacidades políticas en los ciudadanos para la consolidación de una sociedad propositiva. En este sentido, las universidades públicas juegan un papel importante en la construcción del conocimiento e información; así como, en brindar legitimidad técnica para la incidencia política (Collado, 2015).

La UNED tiene como mandato fortalecer los valores en que está fundado el Estado y fomentar el espíritu cívico del pueblo costarricense (Ley № 6044, 1977). Razón por la cual, se crea en la Dirección de Extensión Universitaria la oferta denominada Técnico en Gestión Local. Dicho plan académico pretende proporcionar a los estudiantes capacidades para el involucramiento en el desarrollo del contexto local.

El objetivo del presente documento es sistematizar la experiencia del Técnico en Gestión Local y su contribución al desarrollo de la Región Huetar Norte, durante el año 2017. Dicho documento, se fundamenta en la importancia de aportar recursos cognoscitivos mediante el fortalecimiento de la cultura democrática para la autogestión de un buen vivir en el territorio.

El artículo se organiza en las siguientes secciones: Como antecedentes, se presenta una descripción del plan de estudios y una contextualización del proceso académico llevado a cabo en Upala y San Carlos. Seguidamente, se explica el marco teórico y la metodología empleada. Posteriormente, se aborda el análisis de los aportes del técnico en la construcción de capacidades políticas de los participantes, la elaboración de iniciativas de desarrollo comunal y el inicio de una red de gestores locales.

\section{DESCRIPCIÓN DEL PLAN DE ESTUDIOS DEL TÉCNICO EN GESTIÓN LOCAL}

Esta corresponde a una oferta de educación no formal, la cual procura, mediante la construcción del conocimiento, generar cambios personales en los participantes e incidir en transformaciones sociales. Durante el plan de estudios, se fortalecen las capacidades políticas de los estudiantes para el surgimiento de líderes con bases teóricas y procedimentales que influyan en la gestión de un desarrollo integral y sostenible.

Por tanto, el Técnico en Gestión Local se enmarca en el reconstruccionismo social, donde el conocimiento se genera a partir de la identificación de problemas sociales, el análisis crítico y teorización de los mismos, para, finalmente, buscar soluciones concretas y adaptadas al entorno. Dicha metodología presenta ventajas significativas como lo son la contextualización del proceso de enseñanza-aprendizaje y la puesta en práctica del conocimiento.

Asimismo, el reconstruccionismo social incluye aspectos éticos y socioafectivos; de forma tal, que permite la clarificación de valores y la evaluación de estos respecto a las prácticas, hábitos y costumbres (UNED, 2010). Este aspecto, en un plano de gobierno popular y participación ciudadana, se torna fundamental dado el malestar generalizado de la población debido a hechos de corrupción.

La duración del Técnico en Gestión Local es de un año. Su malla curricular cuenta con seis cursos que involucran tres áreas del conocimiento, las cuales son: 1) desarrollo personal y social; 2) interpretación crítica de la realidad local; 3) procedimientos y estrategias para el desarrollo de la gestión local. Posteriormente, en un lapso de seis meses, se brinda seguimiento al estudiantado en la ejecución de iniciativas de desarrollo local, con el propósito de analizar su desenvolvimiento en la resolución de problemas sociales y facilitar herramientas que permitan un mejor desempeño en su función como gestor local (tabla $1)$. 
Tabla 1: Descripción de la malla curricular del Técnico en Gestión Local y del proceso de seguimiento a los estudiantes

\section{Técnico en Gestión Local}

Duración: 1 año

Cursos

Desarrollo personal y social

Participación en la construcción

de la democracia

Compromiso con el desarrollo

sostenible

Reflexiono acerca del entorno

natural y cultural al que

pertenezco

¿Cómo planificar en el contexto

local?

\section{Seguimiento}

Duración: 6 meses

Áreas del conocimiento Proceso

Desarrollo personal y social, Interpretación crítica de la realidad local

Análisis sobre el

desenvolvimiento

del estudiante en la

ejecución de iniciativas

de desarrollo

Procedimientos y estrategias

para el desarrollo de la gestión local
Asesoría técnica.

Capacitación para

la resolución de los

problemas encontrados

¿Cómo promover la gestión local del desarrollo?

\begin{tabular}{ll}
$\begin{array}{l}\text { Capacidades políticas que se } \\
\text { procuran desarrollar }\end{array}$ & $\begin{array}{l}\text { Actitudinales (saber ser -saber compartir), Conceptuales } \\
\text { (saber teórico) y Procedimentales (saber hacer) }\end{array}$ \\
\hline
\end{tabular}

Fuente: Modificado de Universidad Estatal a Distancia (2010).

\section{CONTEXTUALIZACIÓN DEL TÉCNICO EN GESTIÓN LOCAL EN LA REGIÓN HUETAR NORTE}

El Técnico en Gestión Local en la Región Huetar Norte se implementó en Upala y San Carlos; no obstante, se logró una representación de líderes comunales de San Carlos, Guatuso, Los Chiles, Upala y San Ramón. A la capacitación ingresaron 59 personas, pero lograron culminar exitosamente el proceso 45. El porcentaje de deserción en San Carlos fue de $20 \%$ y en Upala de $31 \%$.

Del total de egresados, 26 son mujeres y 19 son hombres; sin embargo, se logró apreciar una diferenciación de representación por género entre sedes, con una mayoría de hombres en San Carlos (56\%) y una mayoría más marcada de mujeres en Upala (75\%). La representación de la mujer en espacios de toma de decisión es relevante para impulsar procesos de desarrollo equitativos y asegurar el ejercicio de sus derechos.
La edad de los egresados oscila entre los 21 a 67 años, pero el grupo etario más representado es el de los 30 a los 49 años (Tabla 2). Dicha diversidad permitió que durante el proceso de capacitación se realizara un análisis intergeneracional de la gestión del desarrollo en la Región Huetar Norte. No obstante, se vislumbra el reto de continuar formando capacidades políticas en la juventud para asegurar la renovación de liderazgos.

Tabla 2: Número de egresados del Técnico en Gestión Local en la promoción 2017 por rango de edad

\begin{tabular}{|c|c|c|c|}
\hline $\begin{array}{c}\text { Categoría } \\
\text { de edad }\end{array}$ & Upala & San Carlos & Total \\
\hline $\mathbf{2 1 - 2 9}$ & 3 & 4 & 7 \\
\hline $\mathbf{3 0 - 4 9}$ & 12 & 12 & 24 \\
\hline $\mathbf{5 0 - 6 7}$ & 5 & 9 & 14 \\
\hline
\end{tabular}

Fuente: Elaboración propia. 
La diversidad de niveles educativos que caracterizaron a los estudiantes del Técnico en Gestión Local en la Región Huetar Norte hace evidente su cualidad inclusiva, ya que un 5\% no contaba con título de primaria, 38\% tenía primaria completa, 50\% secundaria completa y $7 \%$ poseía un título universitario. No obstante, el grupo de San Carlos presentó un mayor nivel académico en relación con el de Upala.

Dado el carácter multinivel de la capacitación "la expansión de las capacidades de cada participante constituye el propósito fundamental y no la estandarización de los resultados respecto de los aprendizajes" (UNED, 2010, p.14). Razón por la cual, fue indispensable la mediación pedagógica, para partir de los conocimientos previos del estudiante y construir el conocimiento de forma participativa; con el fin de, posteriormente, Ilevarlo a la praxis. Además, surgió la necesidad de adaptar los materiales del curso y la evaluación, puesto que, en algunos casos, debido a las limitaciones encontradas en la expresión escrita, se optó por realizar evaluaciones orales. En adición, se facilitó apoyo extraclase a los estudiantes que así lo requirieran.

\section{ABORDAJE TEÓRICO DE LA GESTIÓN LOCAL}

El objeto del plan académico es la gestión local, entendida como un proceso con múltiples dimensiones, donde están presentes factores situacionales de un territorio e intervienen diversos actores sociales. Dichos actores sociales deben mantenerse en constante interacción y concertación estratégica para el aprovechamiento de las características endógenas de la zona y así alcanzar el mejoramiento de la calidad de vida de la población (UNED, 2010).

Los actores locales son agentes que actúan en relación con cuestiones socialmente problematizadas y son portadores de propuestas que tienden a capitalizar las potencialidades del territorio. Estos están ligados a distintos procesos del desarrollo, tales como: la toma de decisiones, la gestión de aspectos técnicos y la acción local (Chiara, 2004).

Los nuevos modelos de gobernanza establecen el principio de cooperación y colaboración entre actores locales, incluidos dentro de ellos, la ciudadanía; la cual asume un papel de co-decisor de políticas, coproductor de bienes y servicios y contralor de la gestión pública (Oslak, 2013). Sin embargo, para materializar la participación ciudadana, resulta importante la organización social, la cual "se caracteriza por la división del trabajo, la presencia de uno o más centros de poder que controlan los esfuerzos y dirigen hacia los fines" (Etzioni 1975, citado en Cano, 2016, p.8).

Bajo este enfoque, resulta fundamental una construcción colectiva que articule los intereses particulares con el beneficio general. Dichos procesos participativos y de intercambio contribuyen a la construcción de la democracia y dan significado a la política como una práctica social y cultural. Sin embargo:

Dentro de este marco de interacciones, intersubjetividades y producción de sentidos, surgen prácticas de confrontaciones de poder que desencadenan en tensiones y resquebrajamiento social, por las fuerzas de dominación cultural hegemónica, que grupos sociales dominantes imprimen persiguiendo sus intereses. Pero es en la misma cultura, como lugar y construcción colectiva, donde reside la posibilidad para flexibilizar tales tensiones y generar procesos de transformación, donde la interacción simbólica, en el mismo marco intersubjetivo, arroje fórmulas, estrategias y respuestas para la reivindicación y el equilibrio social (Escobar, 2010, p.199).

Entonces, para alcanzar una cultura democrática los ciudadanos deben contar con capacidades que permitan la construcción de un sentido crítico y el cambio de actitudes y comportamientos. De acuerdo con el Programa de las Naciones Unidas para el Desarrollo (2008), el desarrollo de capacidades es el "proceso mediante el cual las personas, organizaciones y sociedades obtienen, fortalecen y mantienen las competencias necesarias para establecer y alcanzar sus propios objetivos de desarrollo a lo largo del tiempo" (p. 4). 


\section{METODOLOGÍA}

La metodología utilizada para la elaboración del documento es la sistematización de experiencias, la cual se adapta al análisis de procesos de intervención social, como el caso del Técnico en Gestión Local. Con este ejercicio de sistematización, se pretende pasar a una "praxis científica", en donde-a partir de la participación en la transformación social—se crea conocimiento que enriquece la acción transformadora y reconoce la cualidad inédita e irrepetible de esta experiencia (Jara, 2012).

Una vez establecido el objeto de la sistematización, se procedió con la definición del eje central, el cual corresponde a la interpretación crítica de los factores que generaron capacidades en los estudiantes para incidir en el desarrollo de la Región Huetar Norte. Posteriormente, se elaboró el plan de sistematización, con el propósito de organizar la información.

Para la recuperación del proceso vivido se utilizaron registros de la experiencia, tales como: informes, bases de datos, minutas de reuniones, memorias de encuentros, fichas de avances de los estudiantes, evaluaciones de los estudiantes a la capacitación, entre otras. Así mismo, se utilizaron fuentes bibliográficas con el propósito de obtener un panorama de investigaciones previas a los temas que se abordan en el documento, pero también para dar un mayor sustento teórico.

\section{DISCUSIÓN}

Como se indicó anteriormente, Costa Rica cuenta con un sistema democrático donde el pueblo tiene poder de decisión en el ámbito electoral, en el administrativo y en el desarrollo local. Sin embargo, para alcanzar un involucramiento real en procesos de toma de decisión, se requiere de capacidades en la ciudadanía que impulsen transformaciones sociales.

Al analizar la experiencia del Técnico en Gestión Local en la Región Huetar Norte en el año 2017, se identificaron los siguientes factores de generación de capacidades para la incidencia del desarrollo en el territorio:
1. Espacios de autoconocimiento y desarrollo socioemocional

La capacitación generó un espacio de autoconocimiento, en el que, mediante introspección, el estudiante identificó sus potencialidades, motivaciones y limitaciones; con el fin de dar sentido a su vida y su interrelación con los demás. Este proceso de reflexión brindó herramientas para la reconstrucción de un plan de vida orientado a cambios que conduzcan al desarrollo personal y social. En la evaluación del plan académico, se rescatan algunas expresiones de estudiantes relacionadas con los aspectos descritos anteriormente:

"Revisar mi vida me dio seguridad, me permitió liberar algunas tensiones y valorar mi experiencia comunal".

"Me ayudó a solucionar dudas e inseguridades personales y como líder comunal".

"Logre demostrar que tengo cualidades que no conocía".

Asimismo, esta experiencia identificó la necesidad de brindar contención emocional a los estudiantes, ya que, en casos particulares, durante el proceso de autoconocimiento surgieron recuerdos negativos que marcaban al individuo. No obstante, cuando la persona logra conocerse a sí misma, se generan atributos como la autovaloración y el empoderamiento.

Según Feixas (1999, citado en la Agencia de la Calidad de la Educación, 2017), "es necesario alfabetizar en las emociones, las habilidades sociales, la toma de decisiones, el manejo de relaciones y en definitiva, en los problemas que afectan de verdad a nuestra vida" (p. 6). Esto debido a que, la presencia de problemáticas socioafectivas reduce la eficiencia del proceso de enseñanza aprendizaje e interfieren en la convivencia.

Con el técnico se concientizó sobre la importancia para el gestor local de crear destrezas sociales que lo faculten al desarrollo de liderazgos positivos. Por ende, se facilitaron técnicas para la comunicación. Respecto a este tema, los beneficiarios expresaron: "aprendí a escuchar y no hacer suposiciones", "he 
logrado tener una muy buena comunicación asertiva, tanto en mi hogar como en los grupos a los que pertenezco", "ahora sé como[sic] hablar en público". Además, algunos estudiantes rescatan el logro de capacidades en el trabajo en equipo, pues indican: "la necesidad de crear equipos de trabajo para mejorar el desarrollo de la organización" y "el curso me ha hecho tomar mayor conciencia sobre la importancia del colectivo".

Como se espera que los graduados del Técnico en Gestión Local sean agentes de cambio en la sociedad, se fortaleció su capacidad de análisis sobre los valores propios y del grupo al cual pertenece. Esto con el fin de propiciar una coherencia de dichos valores con los principios que rigen la sociedad. Según lo expresado por los estudiantes, el técnico propició transformaciones de conducta relacionadas con el respeto, empatía, tolerancia e inclusividad: "he aprendido a valorar a los demás por lo que son", "he aprendido a ponerme en el lugar de otros", "incentivó mi tolerancia", "ahora escucho las opiniones de mis compañeros y las tomo en cuenta".

2. Análisis de la realidad mediante el uso de metodologías educativas que permitan un proceso de enseñanza aprendizaje contextualizado

Un factor clave de éxito en el técnico fue el abordaje metodológico. Este permitió la contextualización del conocimiento mediante la introducción de aspectos epistemológicos, para que, posteriormente, el estudiante comunicara sus ideas, escuchara las ideas de otros y definiera prácticas que se ajusten a su entorno. Esta dinámica utilizó el territorio como recurso pedagógico y permitió aprendizajes significativos al inducir conexiones entre los nuevos aprendizajes y las situaciones del contexto individual del estudiante.

Para acercarse a la realidad, se definieron las características endógenas, las cuales son un factor diferenciador en un entorno globalizado. Los estudiantes hicieron un recuento de recursos naturales y socioculturales presentes en el territorio. Este espacio académico reafirmó la identidad del estudiante, su sentido de pertenencia y reconoció las oportunidades que ofrece su entorno para el desarrollo.
Respecto a este tema, los estudiantes expresaron lo siguiente: "se dio una perspectiva al estudiante de cómo utilizar el recurso cultural y natural en procesos de liderazgo", "me permitió reconocer la realidad de la pérdida de cultura en mi comunidad y la necesidad de volver bajo un enfoque de desarrollo sostenible", "se concientizó en aspectos culturales y los cambios que se deben provocar para traer bienestar a la sociedad", "dio insumos para mejorar la comprensión de mi entorno y poder entender mejor el desarrollo y sus influencias", "el desarrollo debe ser integral, no podemos separar lo económico, social y cultural".

Para la impartición del técnico, el Programa de Gestión Local consideró como criterio de selección la representación de líderes de organizaciones sociales, gobierno local e instituciones, vinculados a temas políticos, educativos, culturales, deportivos, ambientales, productivos (turismo y agricultura), de género y salud. Esta diversidad de actores enriqueció el proceso de aprendizaje mediante el intercambio de conocimientos, experiencias, logros, preocupaciones y problemáticas, lo que facilitó el análisis del desarrollo de la región bajo un enfoque integral.

La interpretación crítica de la realidad permitió al estudiantado identificar y priorizar problemáticas sobre las cuales puede incidir y plantear soluciones con enfoque integral. Sin embargo, en el Técnico en Gestión Local de la Región Huetar Norte hubo casos particulares, donde se identificó dificultad para comprender la multidimensionalidad del desarrollo, lo cual se asocia, sobre todo, a liderazgos tradicionales. Razón por la cual, se diseñaron dinámicas de trabajo en equipo para presentar posiciones con perspectivas más amplias sobre este tema.

Pérez (1994, citado en Melero, 2011) afirma que el análisis de la realidad consiste en acercarse a ella, develarla y conocerla, esto con el propósito de mejorarla. Para ello, se requiere saber: ¿dónde se está?, ¿a dónde se quiere llegar? y ¿cómo hacerlo? Como la pedagogía de los cursos se orienta hacia el fortalecimiento del pensamiento crítico-al valorar la realidad a partir de la información y la reflexión-, se aplicaron metodologías prácticas para la búsqueda 
de información, tales como: técnicas de reconstrucción histórica y procedimientos de investigación-acción participativa. Al respecto, los estudiantes se refirieron como: "aprendí a investigar", "me interesé en conocer más de la historia local de mi comunidad".

\section{Formulación de iniciativas de desarrollo local}

Para la solución eficiente de problemas en el contexto local, se facilitó al estudiantado conocimientos sobre planificación. De forma tal que la acción transformadora tuviera un orden sistemático para el cumplimiento de los objetivos y mayor aprovechamiento de los recursos.

Durante la implementación de la capacitación, se brindaron las herramientas necesarias para completar el ciclo de la planificación (definición de inicia- tivas, formulación de planes, ejecución, monitoreo y evaluación). En relación con ello, los estudiantes expresaron: "al priorizar descubrimos la necesidad comunal", "se debe hacer un diagnóstico primero antes de desarrollar el proyecto", "al planificar el tiempo, horas, recursos humanos, económico, me permite ser más eficiente", "planificar, debe ser parte de nuestra cultura personal y organizacional nada debe quedar al azar".

Durante todo el proceso de enseñanza aprendizaje, se realizó el ejercicio del análisis de la realidad y de planificación del contexto local, lo que dio como resultado la construcción de 31 iniciativas de desarrollo. Estas iniciativas están orientadas a la gestión en temas ambientales, culturales, productivos, educativos, organizativos, de seguridad y de mobiliario e infraestructura.

Gráfico 1: Distribución temática de iniciativas de desarrollo propuestas por los estudiantes del Técnico en Gestión Local en la Región Huetar Norte

\section{Número de iniciativas por temática}

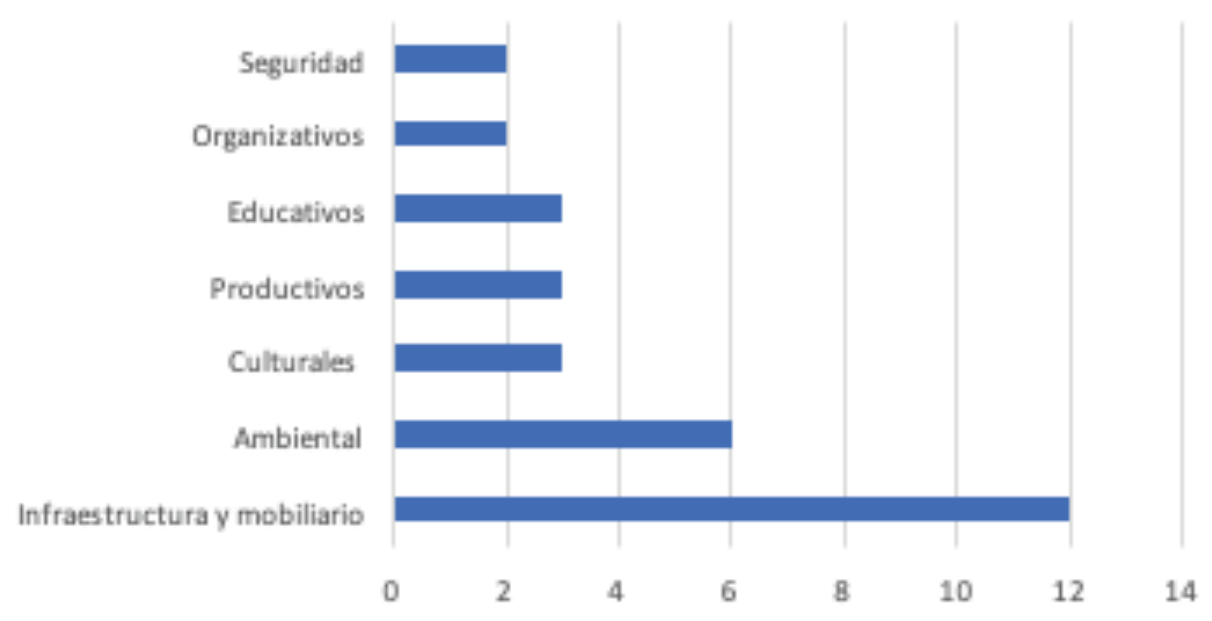

Fuente: Elaboración propia.

El planteamiento de las iniciativas de desarrollo respondió a problemáticas identificadas por los estudiantes en el contexto donde se desenvuelven. Como se observa en el gráfico 1, la mayor cantidad de iniciativas está vinculada con proyectos de infraestructura y mobiliario, entre los cuales: cuatro, se enfocan en infraestructura para la recreación (área deportiva, parque y redondel); tres, en infraestructura de caminos (calles, aceras y parada de buses); dos, en infraestructura de centros vinculados a la salud (CEN CINAI y EBAIS), y tres, en la compra de mobiliario para salones comunales y escuelas. Para su ejecución, será 
importante la articulación con actores institucionales y la gestión de fondos.

Vale la pena mencionar que dos estudiantes de Upala elaboraron una iniciativa de desarrollo cultural denominada "Casa grande", la cual muestra la riqueza natural y cultural de este cantón a partir de expresiones artísticas y fortalece lazos de pertenencia para consolidar una organización comunitaria. Dicha propuesta fue financiada por el Ministerio de Cultura y Juventud.

Además, se concienció a los gestores locales sobre la relevancia de basarse en principios democráticos, donde se involucren diversos actores sociales, institucionales y privados, pero dentro de un marco horizontal de relaciones de poder. De forma tal que se permita el intercambio de necesidades, aspiraciones y preferencias entre los diversos actores para concretar esfuerzos dirigidos al bien común. Respecto a esto, los participantes manifestaron la importancia tanto de la participación comunal para el alcance de objetivos, como de aplicar técnicas para conseguir desarrollar procesos democráticos.

En adición, durante la capacitación, se realizó un análisis crítico de la estructura estatal y los espacios de participación ciudadana con los cuales se dispone. Posteriormente, se plantearon metodologías para tomar decisiones con carácter plural, ya que los líderes comunales representan una diversidad de intereses de personas de la comunidad, incluidas las minorías. Estos elementos, son fundamentales para la incidencia política y la gestión autónoma del desarrollo.

Respecto a este tema, los estudiantes expresaron desarrollar capacidades para la defensa de los derechos y el compromiso para cumplir con sus deberes como ciudadanos. También, indicaron haber logrado una mejor comprensión del concepto de democracia y su papel en la construcción de la misma. Consideran relevante la rendición de cuentas por parte de las organizaciones y formar líderes con conciencia.

4. Identificación de oportunidad de fortalecer redes sociales en la región
Como complemento a la formación técnica, en el año 2017, se llevaron a cabo dos intercambios entre los estudiantes de la Sede de Upala y los de la Sede de San Carlos. El propósito fue establecer relaciones de confianza entre ambos grupos, compartir experiencias y analizar la importancia del trabajo en red.

En dichos espacios, los estudiantes expresaron su interés por conformar una red de gestores locales de la Región Huetar Norte, la cual representa una plataforma para fortalecer su accionar y generar incidencia política. La red que se visualiza es de carácter autogestionario, donde se construyan lazos y pertenencias entre personas que realizan acción social. Por lo tanto, el objetivo inicial de la red es crear un escenario de intercambio de información y experiencias entre los graduados del Técnico en Gestión Local de la Región Huetar Norte.

El principal medio de comunicación de la red será el WhatsApp, razón por la cual se definieron los criterios de participación para su uso. Dada la necesidad de contar con un equipo que lidere esta propuesta, se elaboraron perfiles para los puestos de administradores y enlaces de la red. Seguidamente, se nombraron a representantes del grupo de Upala y San Carlos para que cumplieran estos roles.

Una vez culminado el técnico, los graduados tendrán el reto de consolidar la red como promotor de tejido social. No obstante, se han identificado como limitantes de su desarrollo:

- La falta de claridad y experiencia en el manejo de redes.

- Las personas nombradas como administradores y enlaces de la red no disponen del tiempo necesario para realizar la gestión de la misma.

- Los problemas de acceso a internet de algunos estudiantes, debido a un mal servicio en el lugar donde viven o situaciones económicas que les impiden su pago.

Por su parte, en el ámbito institucional, la vinculación entre el Programa de Gestión Local y el Centro de Investigación, Transferencia de Tecnología y Educación para el Desarrollo (CITTED) durante la 
implementación del técnico en San Carlos fue una innovación, la cual representó una maximización de recursos para la UNED. Además, permitió generar una discusión académica entre ambas dependencias sobre la gestión local del desarrollo en la región y la definición de estrategias educativas para su abordaje.

\section{CONCLUSIONES Y RECOMENDACIONES}

Con la implementación del Técnico en Gestión Local, se identificaron como factores de éxito en la construcción de capacidades para la incidencia del desarrollo en la Región Huetar Norte los siguientes aspectos: la generación de espacios de autoconocimiento y fortalecimiento socioemocional, el análisis de la realidad mediante el uso de metodologías educativas que permitan un proceso de enseñanza aprendizaje contextualizado, la formulación de iniciativas de desarrollo local y la gestión de redes sociales en el territorio.

Con la implementación del Técnico en Gestión Local, se facilitó la construcción de capacidades en los estudiantes, las cuales trascienden al ámbito organizativo y social en el que los participantes se desenvuelven, lo que contribuye al desarrollo de la Región Huetar Norte. Dichas capacidades se categorizan en tres dimensiones:

- Saber teórico: enfoque de desarrollo integral y sostenible, etapas de la planificación, características del liderazgo positivo, cultura democrática, identidad, entre otros.

- Saber hacer: comunicación, resolución de conflictos, trabajo en equipo, análisis e interpretación crítica del contexto, investigación, reconstrucción histórica, planificación, participación ciudadana, elaboración de diagnósticos, formulación de iniciativas del desarrollo, entre otros

- Saber ser: autoestima, seguridad, empoderamiento, respeto, empatía, tolerancia, inclusividad, entre otros.

Una vez finalizado el programa académico se recomienda continuar con el seguimiento a los estudiantes en la ejecución de iniciativas de desarrollo.
Dicho espacio permitirá valorar sus avances y desenvolvimiento en la resolución de problemas sociales. Asimismo, brindará asesoría y capacitación en temas requeridos por el gestor local para mejorar su desempeño.

La consolidación de una red de gestores locales puede significar un catalizador del desarrollo. Por esta razón, se recomienda continuar con este reto y considerar las siguientes necesidades:

- Elaboración de un plan estratégico que oriente su accionar.

- Definición de estrategias de comunicación y vinculación con instancias institucionales.

- Gestión de recursos para su consolidación.

- Trabajo en nodos, los cuales se definen por temática de interés.

- Construcción de una identidad de la red que trascienda las individualidades.

- Fortalecimiento de habilidades comunicativas por medios tecnológicos.

\section{REFERENCIAS BIBLIOGRÁFICAS}

Agencia de Calidad de la Educación. (2017). Indicadores de Desarrollo personal y social en los establecimientos chilenos: una primera mirada [Sitio web]. Recuperado de https://www.supereduc.cl/ estudios-y-estadisticas/los-indicadores-de-desarroIlo-personal-y-social-en-los-establecimientos-educacionales-chilenos-una-primera-mirada/

Alfaro, R. (2012). Cultura política de la democracia en Costa Rica y en las Américas, 2012: Hacia la igualdad de oportunidades [Sitio web]. Recuperado de https://www.vanderbilt.edu/lapop/cr/Costa_Rica_Country_Report_2012_Cover_W.pdf

Cano, D.Y. (2016). Participación ciudadana y gestión del desarrollo local en territorios submunicipales. Estudio de caso: comuna 6-Doce de Octubre de Medellín 2012-2015 (Maestría en Gobierno y Políticas Públicas. Escuela de Humanidades Universidad EAFIT. Medellín). Recuperado 
Fortalecimiento de las capacidades de líderes comunales para la gestión local del desarrollo en la Región Huetar Norte

de https://repository.eafit.edu.co/bitstream/handle/10784/11652/DanyYilban_CanoTorres_2016. pdf? sequence $=2 \&$ isAllowed $=y$

Castro, M. \& Sibaja, G. (2016). Participación ciudadana en democracia: espacios y mecanismos. San José: Instituto de Formación y Estudios en Democracia. Recuperado de http://www.tse.go.cr/pdf/ fasciculos_capacitacion/participacion-ciudadanaen-democracia-espacios-y-mecanismos.pdf

Chiara, M. (2004) La gestión local: Eslabón perdido de las políticas sociales orientadas al desarrollo. Civitas-Revista de Ciências Sociais, 4(2), 353-367.

Collado, H. (2015). Incidencia política, concepto, importancia y herramientas [Sitio web]. Recuperado de http://www.custodiaterritori.org/mm/file/iniciatives/tejiendo\%20redes\%2014-15/Documento\%20incidencia\%20politica_DEF.pdf

Escobar, J.R. (2010). Resignificar la política: Tránsito del sujeto a la acción colectiva [Sitio web]. Recuperado de https://www.files.ethz.ch/isn/126396/m_ culturaipolitica.pdf

Jara, O. (2012). La sistematización de experiencias: práctica y teoría para otros mundos posibles. San José: Centro de Estudios y Publicaciones Alforja.

Ley N ${ }^{\circ}$ 6044. Creación de la Universidad Estatal a Distancia (UNED). Publicada en La Gaceta N50, el 12 de marzo de 1977. Costa Rica.

Ley N 8364. Reforma del párrafo primero del artículo 9 de la Constitución Política de la República de Costa Rica. Publicada en La Gaceta Nº146, el 31 de julio de 2003. Costa Rica.

Melero, N. (2011). El paradigma crítico y los aportes de la investigación acción participativa en la transformación de la realidad social: un análisis desde las ciencias sociales. Cuestiones Pedagógicas, 21, 339-355. Recuperado de https://institucional.us.es/ revistas/cuestiones/21/art_14.pdf

Oslak, O. (mayo, 2013). La gestión pública post NGP en América Latina. Balance y desafíos pendientes. Presentado en la Jornada Inaugural de la IX confe- rencia de la INPAE, Santiago. Chile. Recuperado de http://www.oscaroszlak.org.ar/images/articulosespanol/La\%20Gestion\%20Publica\%20postNGP. pdf

Programa de las Naciones Unidas para el Desarrollo. (2008). Desarrollo de capacidades. PNUD [Sitio web]. Recuperado de http://www.undp.org/content/dam/undp/library/capacity-development/spanish/SP_Nota\%20de\%20Practica_Desarrollo\%20 de\%20Capacidades.pdf

Programa de las Naciones Unidas para el Desarrollo. (2013). Informe nacional sobre desarrollo humano. Aprendiendo a vivir juntos: Convivencia y desarroIlo humano en Costa Rica. PNUD [Sitio web]. Recuperado de http://hdr.undp.org/sites/default/files/ costa_rica_nhdr_2013.pdf

Universidad Estatal a Distancia. (2010). Rediseño del Plan de Estudios para el Técnico en Gestión Local. San José: UNED. 